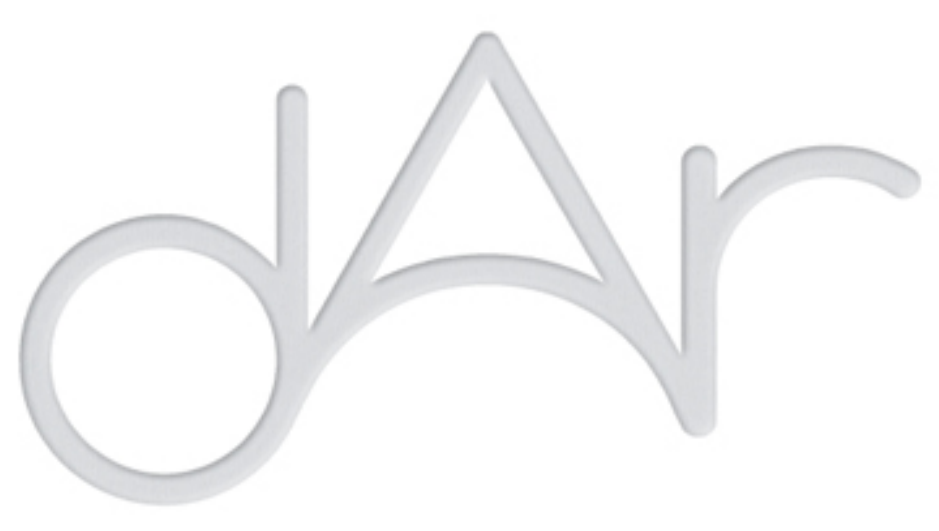

\title{
Quelle mobilisation du patrimoine dans le développement des territoires?: cas l'espace oasien
}

\author{
Autor(es): Jennan, Lahsen \\ Publicado por: Imprensa da Universidade de Coimbra \\ URL \\ persistente: URI:http://hdl.handle.net/10316.2/44424 \\ DOI: $\quad$ DOI:https://doi.org/10.14195/2182-844X_5_3 \\ Accessed : $\quad$ 26-Apr-2023 16:35:50
}

A navegação consulta e descarregamento dos títulos inseridos nas Bibliotecas Digitais UC Digitalis, UC Pombalina e UC Impactum, pressupõem a aceitação plena e sem reservas dos Termos e Condições de Uso destas Bibliotecas Digitais, disponíveis em https://digitalis.uc.pt/pt-pt/termos.

Conforme exposto nos referidos Termos e Condições de Uso, o descarregamento de títulos de acesso restrito requer uma licença válida de autorização devendo o utilizador aceder ao(s) documento(s) a partir de um endereço de IP da instituição detentora da supramencionada licença.

Ao utilizador é apenas permitido o descarregamento para uso pessoal, pelo que o emprego do(s) título(s) descarregado(s) para outro fim, designadamente comercial, carece de autorização do respetivo autor ou editor da obra.

Na medida em que todas as obras da UC Digitalis se encontram protegidas pelo Código do Direito de Autor e Direitos Conexos e demais legislação aplicável, toda a cópia, parcial ou total, deste documento, nos casos em que é legalmente admitida, deverá conter ou fazer-se acompanhar por este aviso.

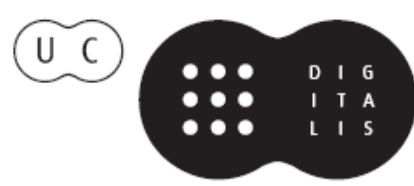



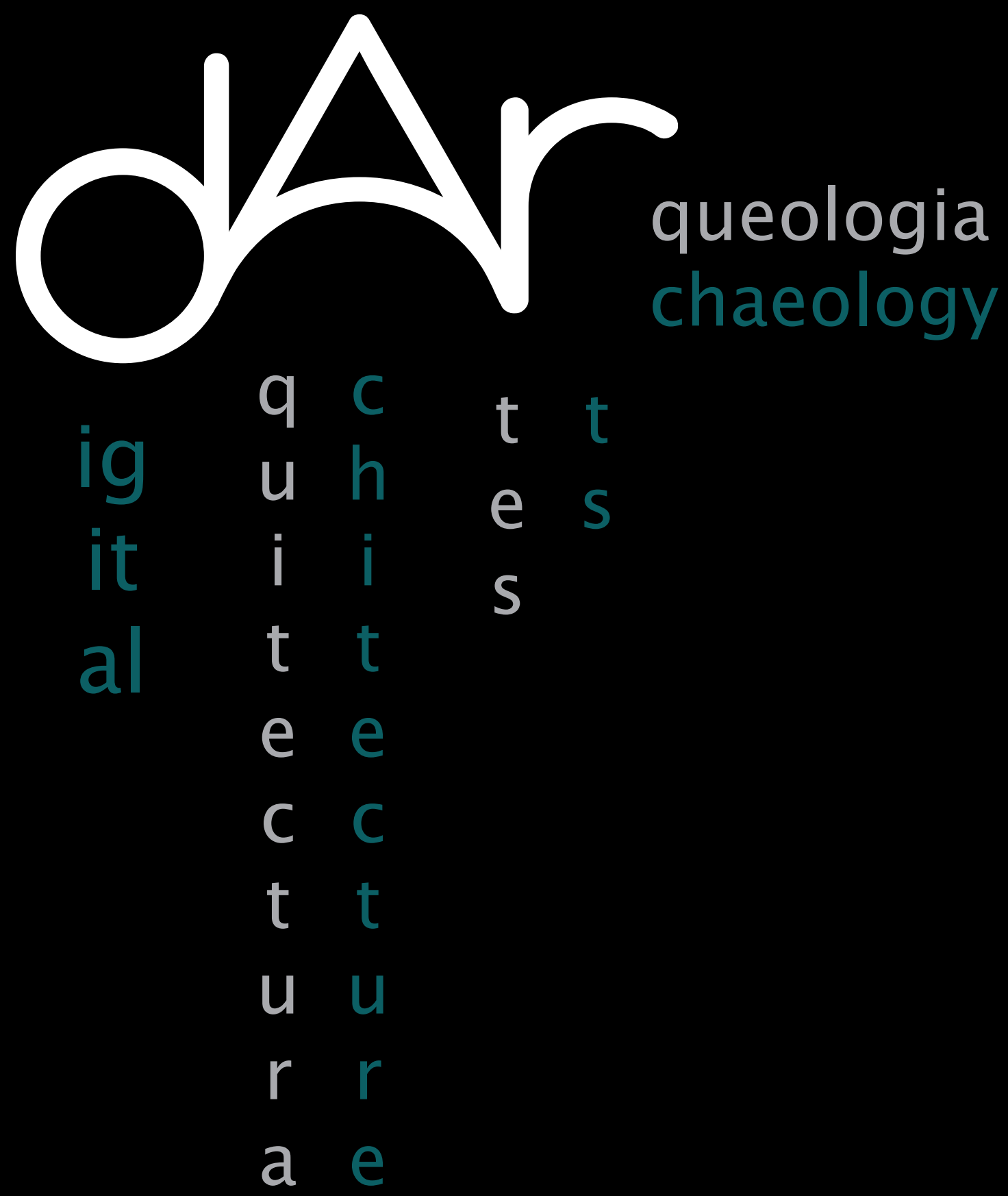

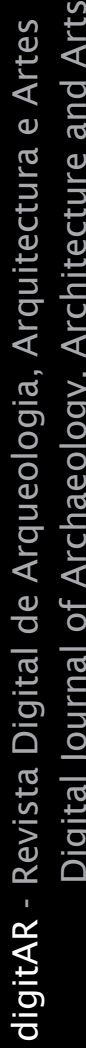




\title{
QUELLE MOBILISATION DU PATRIMOINE DANS LE DÉVELOPPEMENT DES TERRITOIRES ? \\ CAS L'ESPACE OASIEN
}

\author{
Dr. Lahsen Jennan \\ Université de Fés
}

\section{Résumé}

Valoriser ou remettre en valeur des patrimoines, c'est permettre à des territoires de trouver ou de retrouver leur cohérence à travers des références identitaires communes ou l'entretien de liens sociaux à l'occasion d'exercice d'activités communes, notamment symboliques.

Pour construire l'identité d'un territoire, le patrimoine devient porteur de sens et de signification pour la société qui l'a produit, notamment à travers :

L'identification d'un patrimoine commun à l'ensemble du territoire, justifiant d'une identité. La conservation et la restauration d'éléments marqueurs de l'histoire du territoire, permettant de le spécifier par rapport aux autres territoires.

La nouvelle conception du développement local s'appuie sur le fait que tous les espaces ont potentiellement des ressources à condition de $\mathrm{s}^{\prime}$ organiser pour les faire émerger et les valoriser au mieux. Il est ainsi admis que les systèmes territoriaux se caractérisent par la manière dont ils organisent la création et la gestion de leurs ressources.

Enfin, la meilleure façon de conserver le patrimoine, c'est de le valoriser, de lui trouver des usages qui ne portent pas, bien entendu, atteinte à son authenticité. Cette valorisation peut se concevoir dans le cadre d'un projet de territoire dit " pole d'économie du patrimoine » (PEP).

Cette communication se propose d'appliquer la démarche PEP à l'espace oasien en raison du grand potentiel patrimonial que cette aire recèle à l'échelle de la région Draa-Tafilalet.

Mots-clés: patrimoine, oasis, territoire, identité, Draa-Tafilalet 


\section{Introduction}

L'une des conséquences de l'essoufflement du modèle de développement industriel, est l'émergence des procédures de développement local, dont l'une des vocations consiste à protéger et valoriser les patrimoines naturels et culturels des territoires. Acteurs et institutions se sont engagés dans de vastes inventaires des richesses naturelles, mais aussi patrimoniales, en étendant le champ d'étude aux savoir-faire anciens, aux objets vernaculaires, aux traditions et produits de terroir. Leur identification et caractérisation sont le fait de services de l'Etat, mais aussi d'acteurs locaux, en particulier d'associations.

C'est dans ce cadre que s'opèrent les premiers croisements entre patrimoines et territoires. La démarche considère l'usage du patrimoine comme une production de sens qui renseigne à la fois sur les formes de sociabilité et leurs rapports à des formes institutionnelles, politiques et économiques. La construction des territoires et leur (re)composition peuvent être comprises comme un mode d'adaptation à la globalisation. Les acteurs territorialisés tentent d'échapper à l'homogénéisation des règles et à la concurrence par les prix, en engageant des processus de singularisation mobilisant des qualités spécifiques liées aux territoires. En sélectionnant certaines ressources du territoire, en accompagnant leur transmission et leur conservation, en les investissant d'une dimension sociale, culturelle et identitaire, le processus de patrimonialisation va contribuer à les ancrer au territoire et à les élever au statut de ressource territoriale. Cette hypothèse rejoint l'idée que le patrimoine n'est pas une ressource banale, un objet économique commun ; il s'inscrit au cœur de la dynamique territoriale et de son organisation.

Ceci dit, il semble utile de poser d'emblée quelques préalables aux réflexions que nous comptons présenter au lecteur. Ces préalables constituent, en fait, la grille de lecture dans laquelle nous l'invitons à partage ces propos.

En premier lieu, valoriser ou remettre en valeur des patrimoines, c'est permettre à des territoires de trouver ou de retrouver leur cohérence à travers des références identitaires communes ou l'entretien de liens sociaux à l'occasion de l'exercice d’activités communes, notamment symboliques. Ainsi, pour construire l'identité d'un territoire, le patrimoine est/ou devient porteur de sens et de signification pour la société qui l'a produit. Il est porteur des valeurs que souhaitent véhiculer les acteurs de ce territoire, notamment à travers l'identification d'un 
patrimoine commun à l'ensemble du territoire, justifiant d'une identité commune et la conservation d'éléments marqueurs de l'histoire du territoire, permettant de le spécifier par rapport aux autres territoires. D'autre part, la culture populaire, qui est une composante de la culture et du patrimoine en général, ne peut être conçue et mieux appréciée que si elle est considérée et inscrite dans son cadre géographique ou elle a pris forme et au sein d'un contexte patrimonial d'ensemble : il s'agit d'un cadre géographique et d'un mode de vie spécifique d'un système de relations sociales, d'une identité culturelle propre ... etc.

Enfin, la nouvelle conception du développement local s`appuie sur le fait que tous les espaces ont «potentiellement» des ressources à condition de s'organiser pour les faire émerger et les valoriser au mieux. Il est ainsi admis que les systèmes territoriaux se caractérisent par la manière dont ils organisent la création et la gestion de leurs ressources.

\section{La question patrimoniale: contexte et problématique de la valorisation}

\section{1. Le patrimoine face aux défis de la mondialisation}

Face aux défis de la mondialisation, on assiste, de par le monde, à un mouvement de retour au local qui se traduit, pour les communautés, par la recherche d'une réappropriation des origines et la redécouverte des racines, c'est-à-dire d'un renforcement du caractère identitaire et la sauvegarde de la mémoire collective. L'intérêt grandissant porté au patrimoine est ainsi perçu par les sociétés comme un essai de puiser dans les racines (histoire, culture, identité) les éléments de force pour s'adapter à la mondialisation et aménager une place sur l'échiquier mondial. "L'ouverture à la mondialisation se fait d'autant mieux qu' elle prend appui sur de profondes racines et sur une forte identité. S'insérer dans la mondialisation ce n'est donc pas perdre sa différence, mais la cultiver» (Michel Godet, Manuel de prospective stratégique, 1997).

Le retour au local est aussi l'expression d'une utilisation accrue des potentialités endogènes, qui ne signifie nullement un retour au développement local autocentré et autarcique. Au contraire, ce mouvement s'insère dans une dialectique marchandisation/ territorialisation que sous-tendent les mécanismes d'articulation entre mondialisation et développement local. Tout se passe comme si, devant une économie de plus en plus mondialisée, amplifiant la compétition entre territoires, et qui impose la qualification et la mise à niveau des 
territoires et des sociétés, ces dernières s' efforcent de mobiliser et d'insérer leurs ressources propres, et s'investissent au maximum pour assurer leur suivie (économique, culturelle, identitaire) et leur pérennité. Ces ressources territorialisées sont, d'autre part, autant d'objets susceptibles de renforcer les liens de solidarité à l'intérieur du groupe et de lui donner les moyens d'avoir une prise sur les processus qui valident des échelles de temps et d'espace d'un niveau supérieur.

Aujourd'hui, au Maroc, le patrimoine est appelé à devenir, dans le cadre de la régionalisation, de la décentralisation et du développement local, un moteur potentiel de mobilisation des énergies autour d'un sentiment identitaire susceptible de servir de pilier pour la promotion collective et le développement économique et social. Le patrimoine est, en effet, autant un legs du passé qu'un atout du développement. Son intégration dans le processus de développement est une nécessité absolue que sa richesse et sa diversité justifient amplement.

Il constitue un grand potentiel et une immense réserve de richesses qui, loin de nourrir une vision passéiste de la société, des formes et pratiques, est, pour le pays, un atout essentiel de son développement économique et socioculturel actuel et à venir. Tout dépend, en fait, de la manière dont il sera perçu et géré par les décideurs et les acteurs locaux et régionaux. Le patrimoine sera pris ici dans son acception la plus large, tel qu'il est défini par les instances internationales concernées (UNESCO, notamment).

Il regroupe les catégories suivantes :

- le patrimoine monumental classique

- le patrimoine vernaculaire rural

- le patrimoine artisanal et industriel

- les paysages et richesses environnementales

- les identités ethnologiques, savoir-faire, traditions

- les matériaux et ressources spécifiques utilisés dans les activités locales

- la mémoire collective organisée autour d'un fait historique

Seront pris en considération dans cet article, les tes types de patrimoine et leur identification, comme suit :

*Le patrimoine matériel: plus facile à localiser, il se compose de différents éléments:

- Les paysages, qu'ils soient le résultat d'une action séculaire de 
l'homme sur le milieu ou des paysages naturels.

- Les biens immobiliers, qui sont aussi bien les monuments historiques qu'ils soient d'origine militaire, religieuse ou civile (médina, ksours, qasba, remparts, ...) et les bâtiments d'exploitation agricole que ceux liés à l'artisanat, l'industrie, ou à la vie collective et qui témoignent d'activités spécifiques ou tout simplement d'un style architectural, vernaculaire soit-il: village pittoresque, grenier (agadir), khettara, etc.)

- Les biens mobiliers, qui relèvent aussi bien d'usages domestiques que religieux ou festifs.

- Les produits, qui résultent d'une adaptation aux conditions locales et à des traditions de culture, d'élevage, de transformation et de préparation. Ce sont aussi bien des variétés végétales (plantes, fruits, légumes, etc.), des races animales locales (ovin, camelin...) que des produits plus élaborés : artisanat;

* Le patrimoine immatériel : se compose d'un ensemble de biens immatériels qui sont indissociables du patrimoine matériel:

- des techniques et des savoir-faire qui ont permis la création des paysages, la construction de maisons, la fabrication du mobilier, l'élaboration de produits de terroirs ou artisanaux, la construction d'ouvrages hydrauliques, etc... ;

- des parlers locaux, des musiques, une littérature orale, issus de traditions non écrites. Ces modes d'expression témoignent d'une inscription particulière de la communauté sur son territoire et plus généralement d'une façon particulière d'être ensemble. On inclut ici les contes et légendes qui mettent en scène des individus ou des sites qui font partie de l'histoire locale ainsi que les noms des lieux (toponymes) qui reflètent des usages ou des représentations particulières;

- des modes de sociabilité et des formes particulières d'organisation sociale comme certaines coutumes ainsi que des fêtes (religieuses, nationales, calendaires, agricoles, etc.) ou des formes de gestion de la ressource locale.

- Tous ces éléments constituent un patrimoine vivant. Les communautés et les acteurs, en désignant et en s'appropriant ces éléments, leur confèrent un sens pour la collectivité et affirment leur valeur patrimoniale. 


\section{Les déclinaisons de la valorisation du patrimoine}

\section{1. Le patrimoine comme ressource territoriale}

La nouvelle conception du développement local s'appuie, depuis près d'un demi-siècle, sur le fait que les ressources ne sont pas également et uniformément réparties dans l'espace, mais tous les espaces ont " potentiellement» des ressources. Encore faut-il s'entendre sur ce que l'on désigne par ressource.

Au-delà du débat sur la notion de ressource ( qui est au cœur du projet théorique de la pensée économique), il est désormais établi que la question des ressources renvoie à l'articulation entre, d'une part, ces ressources et, d'autre part, les systèmes de production, le territoire et le milieu. Cela veut dire qu'il y aurait des ressources génériques et des ressources directement issues de la dynamique territoriale.

La réflexion autour de la nature des ressources s'est ensuite élargie à la prise en compte des «facteurs latents», c'est-à-dire la possibilité qu'il existe dans toutes les sociétés des ressources non valorisées mais susceptibles de le devenir par le jeu d'effets de proximité, par la formation de dynamiques internes. C'est le cas justement des ressources patrimoniales en milieu oasien et ailleurs.

\section{2. La valorisation du patrimoine ou le passage de la ressource à la création d'activité (Fig. 1).}

Il s'agit du passage du patrimoine de l'état de ressource à celui d'actif susceptible d'être mobilisé. Il n'existe en tant que tel que si la ressource perçue est nantie d'une valeur d'usage. En ce sens, une ressource est une relation sociale, car elle renvoie à l'idée de mise en valeur, aux notions d'échange, de moyens de communication et d'éducation. Le patrimoine qui est un objet transmis, peut être sélectionné pour répondre à des usages. Son statut évolue: d'objet " donné» par héritage, il devient un « bien commun ». Il est en effet non exclusif, en ce sens que le fait de le mobiliser n'empêchera pas d'autres acteurs de l'utiliser pour développer différents usages.

A titre d'exemple, on peut citer les espaces naturels qui, moyennant une organisation intégrant les acteurs des différents usages, vont permettre de promouvoir des actions de protection de la biodiversité, des activités sportives de pleine nature, des actions éducatives, voire de l'exploitation 


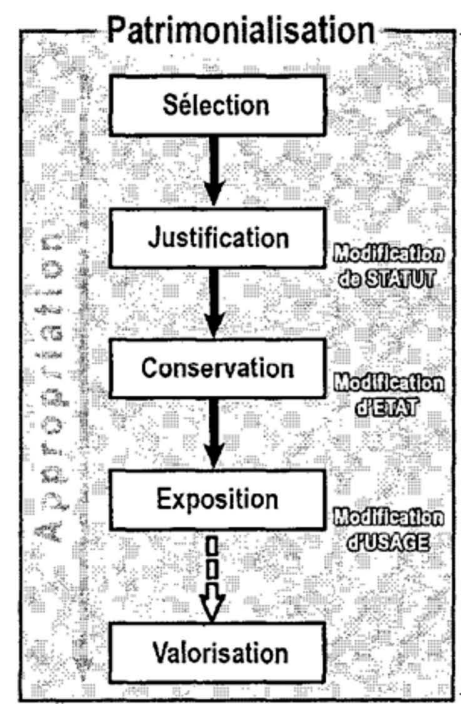

Interprétation

Choix et identification par la collectivité

Classement et octroi d'un statut identitaire

Restauration, réhabilitation, entretien

Passage à l'état d'objet public, de bien

Mobilisation par la création d'activités

Fig. 1 - Les cinq phases du processus de patrimonialisation

forestière. L'espace oasien offre une combinaison différente, en rapport avec ses composantes naturelles, humaines et culturelles propres. Cette activation, qui reste dans le cas du patrimoine optionnelle, est potentiellement créatrice d'activité et d'emplois. Dans ce cas, la ressource patrimoniale valorisée devient source de développement à travers plusieurs itinéraires :

$\checkmark$ En tout premier lieu, le patrimoine fait l'objet d'une réutilisation, permettant le développement d'activités spécifiques.

$\checkmark$ Il peut aussi faire l'objet d'une réaffectation. On relève souvent l'utilisation des bâtis traditionnels pour des activités de restauration, de réception, d'hébergement, voire d'usages publics ; les équipements et événements culturels (festivals, spectacles, résidences d'artistes) ou muséaux, mis en place par des collectivités ou associations, sont souvent développés dans des lieux porteurs d'une valeur patrimoniale.

$\checkmark$ Il peut être mis en réseau, à l'occasion de la mise en place de circuits touristiques et la création de routes à thèmes.

\section{3. Les acteurs de la valorisation du patrimoine}

$\mathrm{Au}$ niveau de chaque Collectivité Locale, les initiatives émanent le plus 
souvent des principaux acteurs du territoire :

- l'administration de tutelle exerce ses prérogatives aux différents échelons du territoire;

- le Conseil Régional peut demander une intervention particulière sur un site phare de la région. Il peut être à l'origine de labellisation;

- le Conseil provincial peut avoir les mêmes ambitions sur son territoire; - les Municipalités et les Communes rurales sont souvent demandeuses d'une valorisation ponctuelle au sein de la Commune (bâtiments ou monuments à valoriser) ou d'une valorisation globale ( plan de mise en valeur d'ensemble);

- les associations de sauvegarde du patrimoine local peuvent sensibiliser les Communes à la dégradation du patrimoine et aux enjeux que sa mise en valeur représente; elles peuvent être, elles mêmes, porteuses de projets ;

- les particuliers, eux aussi, généralement pour leur compte personnel, rénovent et réhabilitent des bâtiments, souvent de caractère (maison distinguées, kasba, ancien corps de ferme, maâsra, moulin, ...) et participent à l'amélioration du patrimoine.

- les organismes scientifiques développent la recherche sur le patrimoine et les modes de sa préservation et de sa valorisation.

\section{La valorisation du patrimoine, un levier du développement des territoires oasiens}

\section{Une alternative adéquate}

L'engouement observé pour le patrimoine naturel ou culturel, s'il est bien géré, peut être une aubaine pour les communes. Il est surtout intéressant de voir en quoi ce phénomène peut aider les collectivités à réorienter leurs activités, notamment vers le tourisme, l'agriculture, l'artisanat et les services induits.

La problématique de la valorisation du patrimoine correspond à une stratégie d'émergence d'une économie du patrimoine dans les territoires concernés.

Un développement optimal prendrait donc en compte les trois éléments fondamentaux du territoire de vie: la société, son économie et sa culture, et décrirait théoriquement une spirale d'interrelations engendrant sans cesse du développement (Fig. 2). 


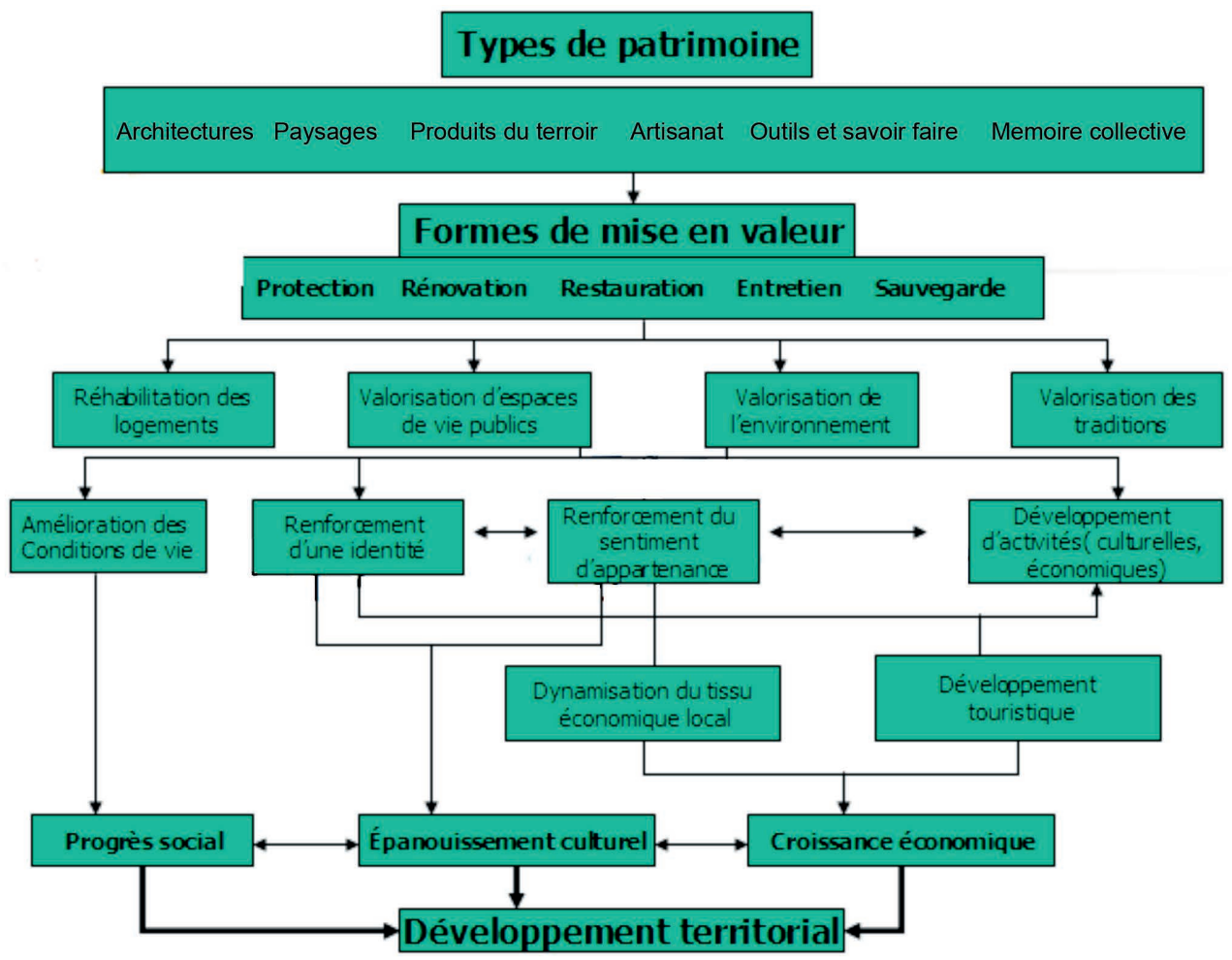

Fig. 2 - De la valorisation du patrimoine au développement territorial

\section{Une démarche constructive}

\section{1. La construction des spécificités.}

D'une part, le processus de développement territorial résulte de la construction de ressources spécifiques au territoire, à travers d'une relation entre des acteurs d'origines internes et externes à ce territoire. D'autre part, la forte intégration des patrimoines dans ce processus permet aux territoires concernées de s'inscrireà la fois dans des logiques de spécificitéet de durabilité, et ce particulièrement dans les marges. En effet, à la suite des géographes qui, au lieu de définir les périphéries seulement comme des espaces dépendant des centres, ils les présentent comme des territoires d'expérimentation porteurs d'innovation, nous considérons en effet que, dans les marges, s'inventent de nouveaux modèles de développement, valorisant des 
qualités attribuées au territoire. Les espaces oasiens constituent un exemple stimulant d'espace marginalisé, non principalement à cause de leurs attributs géographiques, mais d'un enclavement lié à un manque d'infrastructures et à une marginalisation sur le plan socio- économique. Dans ces espaces, la révélation et l'exploitation de nouvelles ressources territoriales constituent une opportunité, un levier de développement économique (favorisant la création d'emplois et de revenus), social (permettant la coordination des acteurs) et identitaire (renforçant l'identification d'un groupe à un territoire).

\section{2. La construction des solidarités.}

Dans un espace présaharien à faible potentiel agricole et vulnérable à maints égards, la démarche de développement à conduire est nécessairement une démarche de développement territorial orientée vers la promotion d'une économie sociale solidaire. Celle- ci doit être sous-tendue par cinq idées forces, véritables piliers de sa stratégie en matière d'actions collectives et de développement social, à savoir :

- le fait de considérer qu'à priori toute population, tout territoire en développement, peut se prendre en charge à partir de ses potentialités, ses ressources, ses capacités, ses savoir-faire et ses solidarités ;

- les initiatives doivent être avant tout collectives et fédératrices et les acteurs capables de négociation à partir de communautés d'intérêts ;

- l'instauration, en vu de son ancrage, d'une visée de promotion collective visant à accroître chez les populations cibles les niveaux de conscience, de la capacité à s'organiser et de la citoyenneté.

- la promotion d'un projet social par le biais de la mise en œuvre d'une stratégie de mieux être social au bénéfice du plus grand nombre ;

- la promotion de territoires de solidarité où œuvrent des partenaires en synergie et interdépendance en vue de construire ensemble, dans une démarche de participation, de négociation et de contractualisation, un projet de territoire correspondant à une stratégie de développement voulue et conçue par l'ensemble des acteurs et de nature à renforcer l'identité territoriale et à créer une dynamique collective autour de la valorisation des ressources spécifiques de ce territoire . 


\subsection{L'émergence d'une nouvelle gouvernance du développement territorial}

Elle correspond au passage d'analyses et de modèles de développement aux échelles infra-nationales ou purement locales vers des analyses multi scalaires. Dans cette nouvelle approche du développement, prenant en compte les acteurs et facteurs du développement à toutes les échelles, le « territoire » est un centre d'intérêt majeur. Il représente alors aussi bien une entité spatiale infranationale dans laquelle est supposé se dérouler le « développement »qu'un concept qui achemine, implicitement ou explicitement, une nouvelle vision, un nouveau modèle de développement : un mode d'adaptation à la mondialisation, au travers de processus de spécification permettant aux acteurs économiques de s'extraire des mécanismes de concurrence par le prix.

Certains territoires se construisent autour de ressources spécifiques, alors que d'autres sont marginalisés, sans spécificités reconnues, dépendant des conditions d'accès aux marchés qui leur sont externes. Ces dynamiques posent la question des nouvelles formes de gouvernance territoriale, et plus particulièrement l'émergence des acteurs associatifs, qui est un fait partagé sur l'ensemble du pourtour méditerranéen. Ce constat amène à interroger le concept $\mathrm{d}$ 'autonomie territoriale, et en particulier de la capacité des acteurs à coordonner des systèmes de normes au service de leurs propres projets.

Cela constitue une profonde modification de la stratégie de développement de ces espaces qui constitue le premier élément et la première innovation de ce qu'on appelle aujourd'hui le développement territorial. Les opérations qui ont permis la mise en $œ u v r e$ de cette valorisation ont par ailleurs rapidement engendré dans ces territoires une nouvelle gouvernance du développement local. Elle consiste notamment à voir le secteur public, les ONG et le secteur privé prendre en charge la gouvernance du développement local. Cette transformation est d'autant plus importante qu'elle provoque une modification forte de la logique d'action de chacun de ces trois acteurs. Ce processus est en émergence au Maroc, en particulier dans sa partie méridionale, dont les oasis, emboîtant ainsi le pas aux anciennes solidarités qui ont par ailleurs facilité cette émergence. L'un des principaux enjeux actuels est la réinvention et l'intégration de ces solidarités dans les processus de développement local. 


\section{Le pôle d'économie du patrimoine (PEP) : une nouvelle conception du développement territorial}

\section{1. Le concept de Pôle d'Economie du Patrimoine (PEP)}

La mise en place des Pôles d'Economie du Patrimoine a pour objectif d'accompagner quelques projets de développement local à partir du patrimoine. L'objectif final est de mettre des lieux et des projets en réseau afin de contribuer au développement de projets de territoire (DATAR, 2001, Pays d'Art et d'histoire). L'idée du réseau sous tend le partenariat entre des acteurs publics et privés, susceptible d'être structuré à partir du patrimoine.

Un PEP n'est pas une structure, ni un label; c'est avant tout une démarche qui prend appui sur les structures existantes, variables selon les cas (pouvoirs publics, collectivités locales, acteurs privés, " pays», régions, associations,, etc. ) et sur la nature et la diversité des éléments patrimoniaux qui caractérisent les territoires de projet considérés (diversité du patrimoine et de la thématique dominante, spécificités des territoires). $C^{\prime}$ est « un processus qui vise à mettre en réseau les initiatives locales en vue de créer du développement économique» (C.Virassamy, 2002, Les pôles d'économie du patrimoine).

Enfin la spécificité d'une démarche PEP tient essentiellement au fait qu'elle dépasse la démarche plus traditionnelle d'élaboration de projets de tourisme culturel pour tendre à la construction d'un projet de développement local intégré, global, cohérent et durable.

\section{2. La démarche PEP}

L'esprit de la démarche «Pôle d'économie du patrimoine » réside dans l'ambition d'identifier les modalités et conditions de construction de projets de territoire qui utilisent le patrimoine non plus comme simples objets culturels et touristiques, mais comme un atout important d'un développement global, innovant en phase avec les attentes contemporaines.

Cette démarche aspire, d'autre part, à doter la ressource patrimoniale d'outils adéquats de promotion lui permettant de dépasser, en terme de développement et de génération de la plus-value économique, le stade de «niche» où il est confiné actuellement pour devenir un potentiel réel de développement pour l'ensemble d'un territoire. 


\section{3. La logique du projet PEP}

Le diagnostic stratégique de l'espace oasien (DAT, 2001, Etude Oasis) permet d'identifier de nombreuses ressources ayant un potentiel de développement réel pour le territoire. Il permet aussi de détecter et de localiser géographiquement les objets patrimoniaux et les ressources spécifiques susceptibles de constituer les produits leaders dans le processus de construction du projet de territoire. Ainsi que l'identification des acteurs locaux (institutionnels, associatifs et privés) impliqués dans les dynamiques de développement local. Il a enfin relevél'existence de différentes dynamiques patrimoniales et de préservation des ressources naturelles à l'œuvre, mais sans coordination réelle et solide entre les projets. Il est ainsi impératif de prendre en compte dans la démarche PEP les nécessités suivantes:

\section{3. 1. La nécessité de fédérer les actions}

Ce qu'il faut, sans équivoque, mettre au profit du territoire oasien est la dynamique patrimoniale déjà engagée autour de projets concrets, déjà à l'œuvre dans lesquels on constate l'existence de trois logiques emboitées:

- Une logique étatique multiforme que portent les différents organes publics régionalisés de l'Etat, exprimés localement en projets sectoriels;

- Une logique de participation en croissance, portée par le tissu associatif et coopératif;

- Des logiques privées, initiées par des acteurs locaux investissant à titre individuel dans des projets de développement.

L'objectif principal du projet de territoire PEP est donc de fédérer l'ensemble des actions et d'impliquer, dès le départ, les acteurs dans une logique de partenariat, de concertation et de solidarité.

\subsubsection{La nécessité d'articuler les ressources aux possibilités de leur valorisation}

Les axes de développement et les actions majeures qui doivent constituer le contenu de ce projet de territoire relèvent évidement de secteurs économiques et sociaux différents mais devront porter, de par leur nature et l'esprit qui devra les animer, les possibilités de mise en réseau entre eux d'abord, et avec d'autres projets intra- territoriaux, ensuite. Qui a -t-il de plus facile à saisir la relation étroite qui associe par exemple un produit de terroir et une offre de site spécifique à un tourisme culturel et écologique ? 
L'objectif du PEP est, par conséquent, d'articuler les actions qui le composent autour d'un ensemble bien déterminé d'enjeux permettant un développement global du territoire. Il s'agit bien de garder à l'esprit les exigences d'un projet global et cohérent organisé autour de l'enjeu touristique, l'enjeu économique et social et l'enjeu environnemental. En effet :

- L'ambition traditionnelle de valorisation du patrimoine s'est toujours réalisée à travers l'activité touristique, exploitant ainsi les objets patrimoniaux dans le cadre de niches. L'enjeu touristique pour le PEP représente, certes, une dimension importante du développement mais ne doit pas être considéré comme une finalité en soi, car le PEP n'est pas un projet touristique au service duquel on doit mobiliser l'ensemble des ressources d'un espace, mais il est bel et bien un projet de territoire.

- Les actions seront donc de nature à promouvoir l'espace de vie des habitants aux plans économique, culturel, social, et environnemental par le développement d'activités multiples : artisanat, agriculture traditionnelle, tourisme, services, formation, préservation des ressources naturelles etc ... et la promotion de ces activités par l'ensemble des acteurs, au profit des populations locales.

\section{3. 3. La nécessité de thématiser les actions}

Le diagnostic territorial doit permettre l'identification des axes stratégiques de développement autour des produits phares, ce qui peut se traduire par une thématisation des actions, chaque axe correspondant, en fait, à un projet spécifique.

Or, dans le cadre du PEP, la thématisation des actions aide à la fédération des projets à l'échelle du territoire. C'est aussi autour d'elle que va se faire la cohésion des différents acteurs et de l'ensemble des partenaires. La thématisation des actions permet de « cimenter » le projet, de construire les passerelles entre les différentes actions et de rendre visible le projet de territoire .

Ceci dit, voici comment, en théorie et dans la pratique, se décline un projet de territoire PEP.

\section{4. Etapes et déclinaisons d'un projet PEP}

La construction d'un projet de territoire PEP passe par trois étapes principales (tableau synoptique ci-après Tab.1) 


\begin{tabular}{|c|c|}
\hline $\begin{array}{c}\text { I - Réalisation d'un diagnostic } \\
\text { stratégique }\end{array}$ & Méthode et outils \\
\hline \multirow{5}{*}{$\begin{array}{l}\text { 1- Inventaire des objets patrimoniaux et } \\
\text { leur répartition géographique (cartographie) } \\
2 \text { - analyse et classification en catégories } \\
\text { selon leur nature, leur potentiel } \\
\text { patrimonialisateur } \\
3 \text { - Identification des espaces à fort et } \\
\text { moyen potentiel patrimonial } \\
4 \text { - Identification et sélection des produits } \\
\text { leaders en fonction de : intérêt économique, } \\
\text { symbole identitaire, degré d'exploitation } \\
\text { actuel, intégration dans le processus de } \\
\text { développement local, valeur d'utilité } \\
\text { collective ou sociale }\end{array}$} & $\begin{array}{l}\text { - Collecte de données stratégiques auprès } \\
\text { des services concernés }\end{array}$ \\
\hline & $\begin{array}{l}\text { - Entretiens semi-directifs auprès des } \\
\text { institutions (administration de tutelle, } \\
\text { province, collectivité locale, association } \\
\text { professionnelle, centres de formation, } \\
\text { leaders d'opinions locaux,...) }\end{array}$ \\
\hline & - Analyse cartographique \\
\hline & - Définition des critères de choix \\
\hline & - Démarche participative \\
\hline
\end{tabular}

\begin{tabular}{|l|l|}
\hline \multicolumn{1}{|c|}{ II - Formulation d'un projet PEP } & \multicolumn{1}{c|}{ Méthode et outils } \\
\hline \multirow{3}{*}{$\begin{array}{l}\text { 1- identification du territoire à ériger en PEP } \\
\text { 2- étude approfondie des ressources } \\
\text { spécifiques : sélection du « panier de } \\
\text { biens et services» }\end{array}$} & $\begin{array}{l}\text { - Entretien auprès des acteurs } \\
\text { sociaux... }\end{array}$ \\
\cline { 2 - 2 } $\begin{array}{l}\text { 3- analyse des modalités techniques et } \\
\text { institutionnelles de sa promotion en PEP }\end{array}$ & $\begin{array}{l}\text { - Démarche participative } \\
\text { synthétique }\end{array}$ \\
\cline { 2 - 2 } & $\begin{array}{l}\text { - Délimitation définitive des lieux de } \\
\text { patrimoine spécifique avec leur mise en } \\
\text { réseau. }\end{array}$ \\
\hline
\end{tabular}

\section{III - Elaboration d'un plan d'action}

1 - Préciser les possibilités de mobilisation et de croisement des acteurs 2 - Proposer des axes de développement d'une économie de patrimoine

3 - examiner les conditions de réalisation de projets articulés

4 - préciser les modalités de préservation des milieux et des patrimoines

La démarche du projet de territoire PEP devant marquer l'articulation entre les différentes composantes du projet est mise en exergue à travers la configuration suivante (voir Tableau 2). L'engagement des partenaires sur un projet commun se concrétise par la signature d'une charte de territoire, signe d'un engagement commun, des partenaires sur un projet à long terme. 


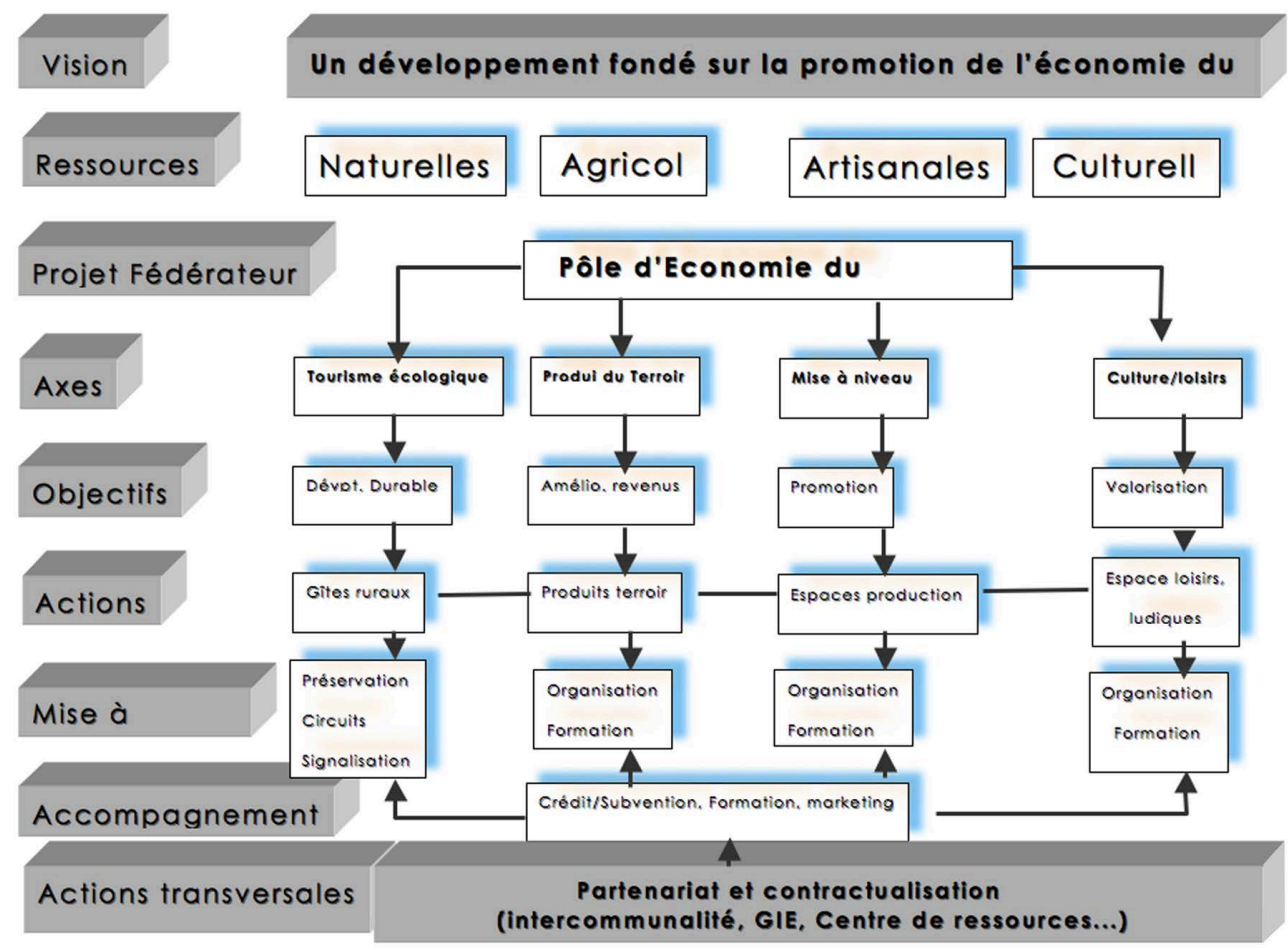

Tab. 2- La consolidation de l'économie du patrimoine des territoires oasiens: pour une « Charte de l'Oasis »

Une charte de territoire n'a pas de valeur réglementaire mais une valeur d'accord moral et politique qui lie les partenaires autour d'un objectif de rang supérieur, dépassant l'intérêt des individus et des institutions.

Elle constitue un document interne au territoire et porte sur des orientations ; elle est différente du contrat, lequel est négocié avec les partenaires extérieurs et se rapporte à un programme d'action.

Nous proposons, dans le cadre de l'approche PEP présentée ci-haut, un projet de charte de l'Oasis visant à créer le cadre et les structures nécessaires où devront s' organiser les acteurs concernés du territoire oasien. Cette charte sera un outil de gestion devant permettre d'organiser les actions et d'engager les acteurs dans une démarche territoriale volontariste de mutualisation des moyens et des compétences, de partage de l'information et d'organisation en réseaux.

Le projet de cette «Charte de l'Oasis » est conçu dans l'esprit et les termes suivants : 


\section{Considérations générales}

- Rappelant la Déclaration Universelle des Droits de l'Homme, adoptée par l'Assemblé Générale des Nations Unies, et les nombreuses déclaration des Nations Unies, tout comme les conventions régionales sur le tourisme, l'environnement, la préservation du patrimoine culturel et le développement durable;

- S'inspirant des principes énoncés dans la Déclaration de Rio sur l'Environnement et le Développement, ainsi que les Recommandations de l'Agenda 2;

- Conscientes que les ressources sur lesquelles se base l'économie oasienne sont fragiles et que la demande pour une meilleure qualité de l'environnement est croissante;

- Estimant que toute action devrait contribuer à faire prendre conscience aux populations oasiennes de l'existence dans leurs territoires d'un patrimoine naturel et culturel unique qu'il convient de sauvegarder pour les générations présentes et futures et conscientes des dangers de destruction que peuvent représenter pour ces territoires une pression exagérée ou certaines pratiques abusives;

- Convaincues que le patrimoine culturel possède une valeur intrinsèque supérieure à sa valeur touristique et conscientes des risque que fait peser sur lui un développement incontrôlé ou excessif et les risques d'uniformisation culturelle et de pertes d'identité qui peuvent en résulter;

- Considérant que, pour participer au développement durable, les activités humaines doivent pleinement s'intégrer dans l'économie locale et contribuer de manière positive à la mobilisation de l'ensemble des ressources locales;

- Considérant que la reconnaissance des acteurs et le soutien de leurs identités, de leur culture et de leurs intérêts doivent être des points de référence incontournables lors de la conception de chaque produit destiné aux territoires oasiens;

- Conscientes de la nécessité de créer un partenariat solide et durable 
entre les principaux acteurs afin de forger l'espoir d'un développement plus responsable vis-à-vis du patrimoine oasien commun;

- Souscrivant aux orientation du Programme de Sauvegarde et Développement des Oasis du Sud du Maroc qui considèrent que le développement touristique n'est pas une fin en soi, mais un levier économique parmi d'autres et que le développement de ce secteur doit, par conséquent, faire partie intégrante d'une démarche territoriale qui unifie toutes les composantes du développement dans une même vision de préservations de la biodiversité et de valorisation au mieux des toutes les ressource locales; Les parties prenantes s'engagent à adhérer aux principes de cette Charte et à œuvrer à la réalisation de ses objectifs tels qu'ils sont définis ci-après:

\section{Principes et objectifs de la charte}

\section{1. Principes}

a - La préservation des territoires et de leurs ressources naturelles (flore, faune, paysages...), culturelles (patrimoine bâti, savoir-faire, identités locales, mémoire collective...) et humains (sociétés sahariennes ou peuples du désert) par le développement d'activités fondées sur:

- le respect de la biodiversité, des éléments naturels et culturels, et de la qualité de l'eau, de l'air, du sol et des paysages;

- le respect de l'identité socio-culturelle des populations locales;

- la responsabilité partagée.

b - La valorisation de l'ensemble des ressources locales actives ou latentes au moyen de produits structurés et d'offre d'un " panier de biens et services » spécifiques et hautement authentique.

c - L'inscription de la logique de ce développement dans une démarche de " patrimonialisation » visant la préservation de la biodiversité et des ressources locales ( approche patrimoniale) par l'usage de techniques douces et la valorisation rationnelle et équitable de ces ressources ( approche économique sociale et solidaire) et générant, à terme, la certification et la labellisation des produits. 


\section{2. Objectifs}

a - La mise en réseau des acteurs locaux en vue de coordonner les actions et de renforcer, via la synergie et l'effort mutuel, les capacités des secteurs et des acteurs à améliorer la qualité de leur offre en terme de produits et de services.

b - La création du cadre nécessaire où devront s'organiser tous les acteurs concernés par le développement oasien durable autour d'une approche commune visant à garantir la qualité et la durabilité des produits et services offerts en milieu oasien.

\section{3- Engagements des parties prenantes (Tab. 3):}

Est considéré comme partie prenante, et peut adhérer à cette Charte, tout groupe ou structure appartenant à l'une des Catégories suivantes :

- acteurs professionnels;

- acteurs institutionnels ;

- populations et société civile.

Ces engagements et mesures sont susceptibles d'être étoffés au désirata des acteurs qui s'y engagent. Ce schéma est à titre indicatif.

Tab. 3 - Six engagements et vingt huit mesures pour un développement oasien durable

\begin{tabular}{|c|c|c|}
\hline & Engagement & Actions/ mesures \\
\hline & $\begin{array}{l}\text { Préserver le patrimoine } \\
\text { naturel et paysager oasien }\end{array}$ & $\begin{array}{l}\text { 1-1: Economiser et rationaliser les ressources en eau } \\
\text { disponibles. } \\
\text { a }- \text { Réaliser des projets touristiques et } \\
\text { agricoles dans les limites des possibilités } \\
\text { hydriques du site; } \\
\text { b - Equiper les structures d'accueil en } \\
\text { économiseurs d'eau et l'agriculture en } \\
\text { système du goutte à goutte. } \\
\text { c- Recycler les eaux usées. } \\
\text { 1-2 : Traiter écologiquement les déchets solides et } \\
\text { liquides. } \\
\text { 1-3 : Protéger les sites et paysages naturels }\end{array}$ \\
\hline
\end{tabular}




\begin{tabular}{|c|c|c|}
\hline & $\begin{array}{l}\text { Construire et agir en } \\
\text { harmonie avec } \\
\text { l'environnement } \\
\text { (conception, style, } \\
\text { matériaux, pratiques } \\
\text { agraires, énergie, } \\
\text { écosystème). }\end{array}$ & 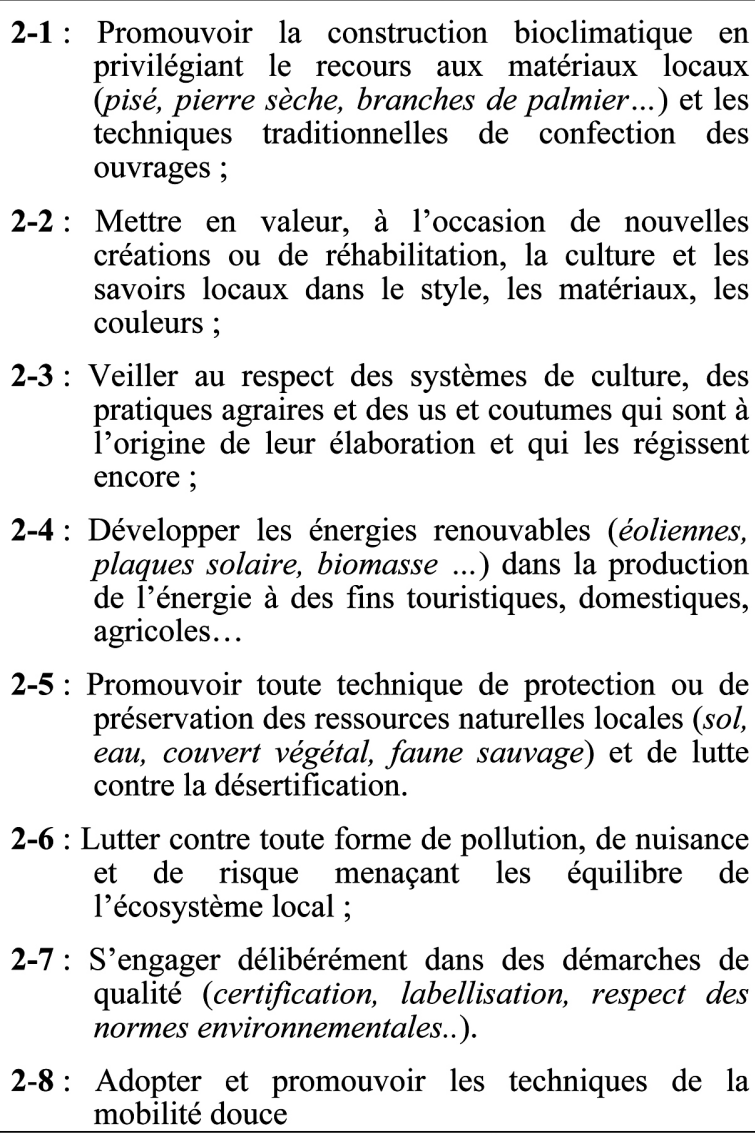 \\
\hline$\checkmark$ & $\begin{array}{l}\text { Protéger et valoriser } \\
\text { l'identitaire et les savoirs } \\
\text { et savoir-faire locaux }\end{array}$ & 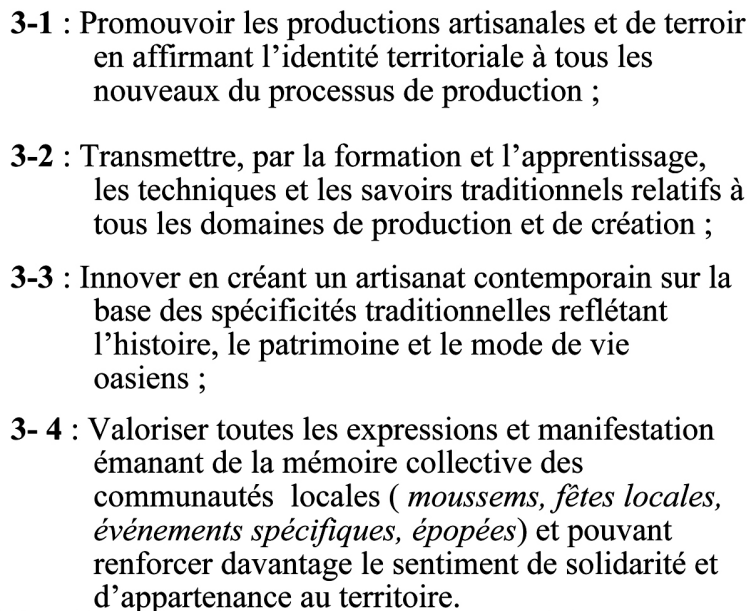 \\
\hline
\end{tabular}




\begin{tabular}{|c|c|c|}
\hline & $\begin{array}{l}\text { Développer le territoire } \\
\text { Oasien }\end{array}$ & $\begin{array}{l}\text { 4-1 : Articuler la production locale aux besoins du } \\
\text { tourisme local; } \\
\text { 4-2 : Garantir la provenance oasienne /locale des } \\
\text { produits destinés aux touristes ; } \\
\text { 4-3 : Garantir l'authenticité et la qualité de ces produits ; } \\
\text { 4-4 : Cultiver bio, diversifier l'offre et améliorer les } \\
\text { techniques de production; } \\
\text { 4-5 : Soutenir l'organisation en groupement de } \\
\text { production (coopératives, associations) pour une } \\
\text { meilleure qualification des produits ; } \\
\text { 4-6 : Encourager tout mode de production, de protection } \\
\text { des ressources, d'organisation ou de gestion } \\
\text { (partenariat, innovation, success story, recherche } \\
\text {..) pouvant générer croissance économique et } \\
\text { progrès social et pouvant contribuer au } \\
\text { développement territorial du site } \\
\text { 4-7 : Apporter le soutien nécessaire à l'organisation des } \\
\text { filières touristiques, artisanales, agro-alimentaires, } \\
\text { culturelles et artistiques locales et favoriser leur } \\
\text { mise en réseau autour d'activités intégrées de } \\
\text { développement territorial. }\end{array}$ \\
\hline & $\begin{array}{l}\text { Connaître et promouvoir } \\
\text { les qualités distinctives du } \\
\text { site. }\end{array}$ & $\begin{array}{l}\text { 5-1 : Dresser un inventaire renouvelé des atouts du site ; } \\
\text { 5-2 : Dresser un inventaire des savoir -faire locaux et de } \\
\text { leurs détenteurs dans tous les domaines de } \\
\text { l'activité humaine; } \\
\text { 5-3 : Soutenir les efforts de recherche scientifique sur } \\
\text { toutes les composantes de l'espace et de la société } \\
\text { oasiens; } \\
\text { 5-4 : Composer et diffuser des outils pédagogiques } \\
\text { divers sur le site en vue d'en accroître la } \\
\text { connaissance et l'attractivité. }\end{array}$ \\
\hline & $\begin{array}{l}\text { Promouvoir une démarche } \\
\text { soutenue de renforcement } \\
\text { des capacités }\end{array}$ & $\begin{array}{l}\text { 6-1 : Dispenser de façon régulière des sessions de } \\
\text { formation spécifiques (thématiques ou } \\
\text { composées) aux profits des différentes catégories } \\
\text { d'acteurs; } \\
\text { 6-2 : Soutenir la formation aux métiers du tourisme, du } \\
\text { patrimoine et de l'environnement, en relation } \\
\text { avec les spécificités et problématiques locales. }\end{array}$ \\
\hline
\end{tabular}




\section{Des mesures d'accompagnement}

4. 1. Créer une structure intersectorielle de concertation et de coordination, pour mettre en place et assurer le suivi d'un mécanisme de coopération et de partenariat entre gestionnaires du territoire, agriculteurs, artisans, ONG, acteurs culturels et touristiques . Ce comité de pilotage peut être constitué au niveau de la commune (rurale, urbaine) ou d'une hiérarchie administrative supérieure.

4. 2. Développer des partenariats à long terme avec les associations et ONG du territoire en tant qu'organismes de proximité et facilitateurs en tout genre (connaissance des acteurs et des dynamiques culturelles, sociales et économiques locales, de la démarche participative, des techniques de négociation et de contractualisation, des réseaux et modes de financement des projets ... etc.).

4. 3. Proposer et soumettre aux partenaires, pour concertation et adoption, toute autre forme d'organisation et/ou de mobilisation des acteurs pour la mise en œuvre de cette Charte.

\section{Conclusion}

Le développement par le patrimoine n'est pas une démarche de " compensation » visant à remédier aux échecs des autres secteurs économiques. Le PEP est un axe novateur du développement territorial basé sur une vision contemporaine du patrimoine, tournée vers l'avenir et en rupture avec les discours empreints de nostalgie et de passéisme dont le patrimoine a trop longtemps pati. Le patrimoine est une ressource de développement durable. Sa valorisation peut constituer un atout indéniable du développement local dans les zones défavorisées ou en quête de nouveaux potentiels de croissance.

Toutefois, la valorisation du patrimoine, en raison des statuts variés et souvent complexes de celui-ci, impose la résolution à l'amont d'un certain nombre de problèmes pouvant provenir des statuts ou des usages dont on compte doter les éléments patrimoniaux objet de cette valorisation.

En effet, la " patrimonialisation » d'un bien ou d'un savoir réside dans le fait de lui donner du « sens ».Ce bien ou ce savoir ne peut être considéré en soi comme un élément patrimonial. Elle fait d'un bien particulier un « bien commun » ayant une valeur collective potentielle. Elle institue un type de 
lien spécifique d'appropriation entre un élément donné et des personnes n'ayant pas de lien juridique avec lui. Dès lors, l'usage de ce bien suppose l'élaboration d'un consensus entre les différents utilisateurs potentiels. Ces derniers peuvent avoir entre eux des conceptions différentes de l'usage possible. Elle impose, par conséquent, une démarche participative .

A moins de supposer que le problème se règle par une démarche de nature juridique du type expropriation, la seule voie possible est celle de la négociation entre acteurs concernés.

Pour éviter que ne surviennent sans cesse de nouvelles tensions ou antagonismes sur les enjeux d'une " patrimonialisation », il est indispensable, dès le début de la réflexion sur l'utilisation d'un élément patrimonial, d'associer le plus grand nombre d'acteurs potentiellement concernés et de mobiliser le maximum de connaissances sur les usages possibles.

\section{Bibliographie}

CULture, CULtURE TERRITORIALE, PATRIMOINE ET TERRITOIRES.

BARRERE C., BARTHELEMY D., NIEDDU M., VIVIEN F-D ? (2004) : Réinventer le patrimoine, de la culture à l'économie, une nouvelle pensée du patrimoine, Paris, L'Harmattan.

BAYARD J.F., L'illusion identitaire, Fayard, Paris, 306 p.

BERARD L., MARCHENAY P., (2004), Les produits de terroir, entre cultures et règlements, CNRS éditions, Paris, $229 \mathrm{p}$.

CLAVAL P. (1995), Géographie culturelle, Coll. Fac Géographie, Editions Nathan Université, Paris, $384 \mathrm{p}$.

CERMOSEM (2002).Patrimoines, territoires et création d'activités. Montagnes Méditerranéennes n¹5, IGA, Grenoble.

COLLECTIF (1996) : Le territoire, géographie et cultures, L'Harmattan, Paris,143 p. CUCHE D. (1996) : La notion de culture dans les sciences sociales, Editions La Découverte, Paris, 124p.

HIRCZAK M., PECQUEUR B., MOLLARD A., (2004), «Le panier de biens et de services de qualité : vers un modèle de développement territorial durable», Montagnes Méditerranéennes, $\mathrm{n}^{\circ} 20$, pp.35-42.

JENNAN L. (sous la direction de).2007. Patrimoines. Territoires et développement. Cahiers Géographiques n³/4, PATER, Faculté des Lettres et Sciences Humaines DM, Fès, 240p 
BARRERE C et al. (2005). Repenser la catégorie économique de patrimoine ? Géo. Econ. et Soci. Vol : 6, n³, pp237-242.

GUMUCHIAN H et PECQUEUR B, (2007). La ressource territoriale. Ed.

Economica, 248p.

PEYRACHE GADEAU V., PECQUEUR B. (2004) : Les ressources patrimoniales, valorisation par les milieux innovateurs ; dans Ressources naturelles et culturelles, milieux et développement local, GREMI, Neuchâtel, Editions EDES, 298 p.

MATTEACCIOLI A. (2004) : Philippe AYDALOT, pionnier de l'économie territoriale, L'Harmattan, Théorie sociale contemporaine, $413 \mathrm{p}$.

TROIN J F (2002). Maroc. Régions, pays et territoires. Ed. Maisonneuve et Larose.

\section{RECHERCHE ACTION ET TERRITOIRES}

Direction de l'Aménagement du Territoire, (2001). Stratégie d'aménagement et de développement des Oasis au Maroc. Marché n6/2001

GLEVAREC $\mathrm{H}$ et SAEZ G (2002).Le patrimoine saisi par les associations. La Documentation Française, Paris

HIRCZAK F et SENIL N (2005). Diagnostic de territoire et ressource territoriale, apports croisés et opérationnalité. IREGE-EDITEM, Annecy.

JENNAN L, LANDEL P A et SENIL N(2007). Le patrimoine : une ressource pour le développement territorial. Expériences de mise en œuvre de pôles d'économie du patrimoine au Maroc. Rev. Cahiers Géographiques n³-4 . Pater, FLSH/DH, Univ. de Fès, pp.11-19.

Ministère de la Culture et de la Communication -DATAR, (2001). Pays d'Art et d'Histoire. La documentation Française. Paris.

VIRASSAMY C, (2002). Les Pôles d'économie du patrimoine. Documentation Française, Datar. Paris. 89p. 